\title{
Teleportation via maximally and non-maximally entangled mixed states
}

\author{
Satyabrata Adhikari* \\ Dept. of Mathematics, Birla Institute of Technology, Mesra, Ranchi-835215, India \\ Archan S. Majumdar \\ Dept. of Astro Phys. and Cosmology, S. N. Bose National Centre of Basic Sciences, Salt lake, Kolkata-700098, India \\ Sovik Roy ${ }^{\ddagger}$ \\ Dept. of Mathematics, Techno India, EM 4/1-Salt Lake City, Kolkata-700091, India \\ Biplab Ghosh $\S$ \\ Dept. of Phys., Vivekananda College for Women, Barisha, Kolkata 700008, India \\ Nilkantha Nayak \\ Centre for Applied Physics, Central Univ. of Jharkhand, 835205, India
}

(Dated: October 10, 2018)

\begin{abstract}
We study the efficiency of two-qubit mixed entangled states as resources for quantum teleportation. We first consider two maximally entangled mixed states, viz., the Werner state 1], and a class of states introduced by Munro et al. 22. We show that the Werner state when used as teleportation channel, gives rise to better average teleportation fidelity compared to the latter class of states for any finite value of mixedness. We then introduce a non-maximally entangled mixed state obtained as a convex combination of a two-qubit entangled mixed state and a two-qubit separable mixed state. It is shown that such a teleportation channel can outperform another non-maximally entangled channel, viz., the Werner derivative for a certain range of mixedness. Further, there exists a range of parameter values where the former state satisfies a Bell-CHSH type inequality and still performs better as a teleportation channel compared to the Werner derivative even though the latter violates the inequality.
\end{abstract}

PACS numbers: 03.67.-a, 03.67.Bg, 03.65.Ud

\section{INTRODUCTION}

Quantum teleportation is one of the most relevant applications of quantum information processing. Teleportation requires the separation of a protocol into classical and quantum parts using which it is possible to reconstruct an unknown input state with perfect fidelity at another location while destroying the original copy. The original idea of teleportation introduced by Bennett et al. 3] is implemented through a channel involving a pair of particles in a Bell State shared by the sender and the receiver. Later, Popescu [4] showed that pairs in a mixed state could be used for imperfect teleportation. Further, it has been shown that if the two distant parties adopt a "measure-and-prepare" strategy for teleporting an unknown quantum state, then the average fidelity of teleportation is at most $2 / 3$ which is the maximum fidelity achievable by means of local operations and classical communications [4] 6 . A quantum channel could be useful for communication purposes only if its teleportation fidelity exceeds 2/3.

In practice it is difficult to prepare pure states, but rather the states obtained are generally mixed in their characteristics. Naturally, a question arises as to whether better average teleportation fidelities compared to that in classical protocols could be obtained if mixed states were used in quantum communication purposes. Therefore, the basic objective is to look for such mixed states which when used as quantum teleportation channels, give fidelity of teleportation higher than the classical fidelity 2/3. It has been found that Werner states [1] used as quantum teleportation channels give higher teleportation fidelity [7]. Recently, the mixed state obtained from the Buzek-Hillery cloning machine 8 as a teleportation channel has been studied [9].

\footnotetext{
*tapisatya@gmail.com

†archan@bose.res.in

¥sovik1891@gmail.com

§quantumroshni@gmail.com
} 
Similar to the case of pure entangled states, entangled mixed states can also be divided into two categories: (i) maximally entangled mixed states (MEMS) and (ii) non-maximally entangled mixed states (NMEMS). Those states that achieve the greatest possible entanglement for a given mixedness are known as MEMS, otherwise they are NMEMS. The notion of MEMS was first introduced by Ishizaka and Hiroshima 10. They proposed a class of bipartite mixed states and showed that entanglement of those states cannot be increased further by any unitary operations (e.g., the Werner state). Later, Munro et.al. (MJWK) 2] studied a class of states which has the maximum amount of entanglement for a given degree of purity and derived an analytical form for that class of MEMS. Apart from maximally entangled mixed states, there are also NMEMS which can be studied for some particular interest. Hiroshima and Ishizaka [1] studied a NMEMS called Werner derivative which can be obtained by applying a unitary transformation on the Werner state.

The motivation for this work lies in performing a comparitive study of mixed states in their capacity to perform as efficient channels for quantum teleportation. It is known that not all entangled mixed states are useful for teleportation [16. So the question arises as to whether all MEMS states could be used as teleportation channels. To this end we first explore the capability of the MJWK class of states 2] as teleportation channels by finding their average teleportation fidelity. We find an upper bound for mixedness beyond which the MJKW class of states is not useful for teleportation. We further show that Werner states always act as better teleportation channels for all finite values of mixedness, even though they are less entangled compared to MJWK states for a given entropy. We then focus on non-maximally entangled mixed states and probe a question: is there any family of NMEMS which outperforms existing NMEMS such as the Werner derivative states[11] when used for quantum communication purposes ? To address this issue we construct a new entangled mixed state which is the convex combination of an entangled mixed state and a separable mixed state. Our state is NMEMS since it does not fall in the class of Ishizaka and Hiroshima's [10] MEMS. We show that this class of NMEMS can serve better as quantum channel for teleportation compared to the Werner derivative for a range of values of mixedness.

The relation between nonlocality of states as manifested by the violation of Bell-CHSH inequalities [12] and their ability to perform as efficient teleportation channels is interesting. It has been shown that there exist mixed states that do not violate any Bell-CHSH inequality, but still can be used for teleportation[4. Here we raise this question first with regard to MEMS states and show there exists states in this category which satisfy the Bell-CHSH inequality, but could be still useful for teleportation. We then consider NMEMS states and find a range of parameters for which our constructed state satisfies a Bell-CHSH type inequality but still outperforms the Werner derivative in teleportation, even though the latter violates the Bell-CHSH inequality. Finally, our comparitive study of teleportation by maximally and non-maximally entangled mixed states reveals that whereas in the former case, one class of states, i.e., Werner states, definitely outperforms another, i.e., MJWK states for all values of mixedness, the result for the NMEMS states that we consider depends on their degree of mixedness.

The paper is organized as follows. In section-II, we recapitulate some useful definitions and general results related to mixed states, their violation of local inequalities, and the optimal teleportation fidelities when they are used as teleportation channels. We illustrate these general results with the well-known example of the Werner state [13]. In section-III, we study the efficiency of the MJWK states [2] in teleportation. We then consider two different NMEMS in Section-IV. We first study the Werner derivative[11] as a teleportation channel and also obtain the range of parameter values for which it violates the Bell-CHSH inequality. We next introduce another NMEMS and investigate its entanglement properties and efficiency as a teleportation channel. We further show that this new NMEMS satisfies the Bell-CHSH inequality. In Section-V we present a comparitive analysis of the MEMS as well as the NMEMS channels for teleportation, also highlighting their respective status vis-a-vis the Bell-CHSH inequality. Finally, we summarize our results in Section-VI.

\section{THE WERNER STATE AS A TELEPORTATION CHANNEL}

The Werner state is a convex combination of a pure maximally entangled state and a maximally mixed state. Ishizaka and Hiroshima [10] showed that the entanglement of formation [14] of the Werner state cannot be increased by any unitary transformation. Therefore, the Werner state can be regarded as a maximally entangled mixed state. In this section we will review the performance of the Werner state as a teleportation channel. Though most of the results presented here are well known [13, our discussion is intended to set the stage for the analysis of other MEMS and NMEMS states that we perform later. To begin with, let us recall certain useful definitions on the entanglement, teleportation capacity and mixedness of general states.

The maximal singlet fraction is defined for a general state $\rho$ as [15]

$$
F(\rho)=\max \langle\Psi|\rho| \Psi\rangle
$$

where the maximum is taken over all maximally entangled states $|\Psi\rangle$. 
The linear entropy $S_{L}$ for a mixed state $\rho$ is defined by [2]

$$
S_{L}=\frac{4}{3}\left(1-\operatorname{Tr}\left(\rho^{2}\right)\right)
$$

The concurrence for a bipartite state $\rho_{A B}$ is defined as 14

$$
C=\max \left\{0, \lambda_{1}-\lambda_{2}-\lambda_{3}-\lambda_{4}\right\}
$$

where $\lambda$ 's are the square root of eigenvalues of $\rho \tilde{\rho}$ in decreasing order. The spin-flipped density matrix $\tilde{\rho}$ is defined as

$$
\tilde{\rho}=\left(\sigma_{y}^{A} \otimes \sigma_{y}^{B}\right) \rho^{*}\left(\sigma_{y}^{A} \otimes \sigma_{y}^{B}\right)
$$

The efficiency of a quantum channel used for teleportation is measured in terms of its average teleportation fidelity given by 18 ]

$$
f_{o p t}^{T}\left(\rho_{\phi}\right)=\int_{S} d M(\phi) \sum_{k} p_{k} \operatorname{Tr}\left(\rho_{k} \rho_{\phi}\right)
$$

where $\rho_{\phi}$ is the input pure state and $\rho_{k}$ is the output state provided the outcome $k$ is obtained by Alice. The quantity $\operatorname{Tr}\left(\rho_{k} \rho_{\phi}\right)$ which is a measure of how the resulting state is similar to the input one, is averaged over the probabilities of outcomes $p_{k}$, and then over all possible input states ( $M$ denotes the uniform distribution on the Bloch sphere $S$ ). It has been shown [16] that if a state is useful for standard teleportation, the optimal teleportation fidelity can be expressed as

$$
f_{o p t}^{T}(\rho)=\frac{1}{2}\left[1+\frac{N(\rho)}{3}\right]
$$

where $N(\rho)=\sum_{i=1}^{3} \sqrt{u_{i}}$ and $u_{i}$ 's are the eigenvalues of the matrix $T^{\dagger} T$. The elements of the matrix $T$ are given by

$$
t_{n m}=\operatorname{Tr}\left(\rho \sigma_{n} \bigotimes \sigma_{m}\right)
$$

where $\sigma_{i}$ 's denote the Pauli spin matrices. Now, in terms of the quantity $N(\rho)$, a general result 16 holds that any mixed spin- $\frac{1}{2}$ state is useful for (standard) teleportation if and only if

$$
N(\rho)>1
$$

The relation between the optimal teleportation fidelity $f_{o p t}^{T}(\rho)$ and the maximal singlet fraction $F(\rho)$ is given by [7]

$$
f_{\text {opt }}^{T}(\rho)=\frac{2 F(\rho)+1}{3}
$$

From Eqs. (6) and (9) it follows that

$$
F(\rho)=\frac{1+N(\rho)}{4}
$$

Now using the inequality [17]

$$
F \leq \frac{1+N}{2} \leq \frac{1+C}{2}
$$

where $N$ denotes the negativity of the state, we have

$$
N(\rho) \leq 1+2 N
$$

We now recall a useful result on the the violation of the Bell-CHSH inequality by mixed states. Any state described by the density operator $\rho$ violates the Bell-CHSH inequality [12] if and only if the inequality

$$
M(\rho)=\max _{i>j}\left(u_{i}+u_{j}\right)>1
$$

holds, where $u_{i}$ 's are eigenvalues of the matrix $T^{\dagger} T[16$. 
Let us now review the Werner state as a resource for teleportation [13. Though the Werner state can be represented in various ways, in the present work we express it in terms of the maximal singlet fraction. The Werner state can be written in the form

$$
\begin{aligned}
\rho_{W}= & \frac{1-F_{w}}{3} I_{4}+\frac{4 F_{w}-1}{3}\left|\Psi^{-}\right\rangle\left\langle\Psi^{-}\right| \\
= & \left(\begin{array}{cccc}
\frac{1-F_{w}}{3} & 0 & 0 & 0 \\
0 & \frac{1+2 F_{w}}{6} & \frac{1-4 F_{w}}{6} & 0 \\
0 & \frac{1-4 F_{w}}{6} & \frac{1+2 F_{w}}{6} & 0 \\
0 & 0 & 0 & \frac{1-F_{w}}{3}
\end{array}\right)
\end{aligned}
$$

where $\left|\Psi^{-}\right\rangle=\frac{|01\rangle-|10\rangle}{\sqrt{2}}$ is the singlet state and $F_{w}$ is the maximal singlet fraction corresponding to the Werner state. $F_{w}$ is also related to the linear entropy $S_{L}$ as

$$
F_{w}=\frac{1+3 \sqrt{1-S_{L}}}{4}
$$

The concurrence of $\rho_{W}$ is given by

$$
\begin{aligned}
C\left(\rho_{W}\right) & =\max \left\{0,2 F_{w}-1\right\} \\
& =\left\{\begin{array}{cc}
0 & 0 \leq F_{w} \leq \frac{1}{2} \\
2 F_{w}-1 & \frac{1}{2}<F_{w} \leq 1
\end{array}\right.
\end{aligned}
$$

When the Werner state is used as a quantum channel for teleportation, the average optimal teleportation fidelity is given by $7,19,20$

$$
f_{o p t}^{T}\left(\rho_{W}\right)=\frac{2 F_{w}+1}{3}, \quad \frac{1}{2}<F_{w} \leq 1
$$

Similarly, the relation between the teleportation fidelity and the concurrence of the Werner state is given by

$$
f_{o p t}^{T}\left(\rho_{W}\right)=\frac{2+C\left(\rho_{W}\right)}{3}
$$

In terms of the linear entropy $S_{L}$, Eq. 17) can be re-written as

$$
f_{o p t}^{T}\left(\rho_{W}\right)=\frac{1+\sqrt{1-S_{L}}}{2}, \quad 0 \leq S_{L}<\frac{8}{9}
$$

Further, we have

$$
F\left(\rho_{W}\right)=\frac{1+N\left(\rho_{W}\right)}{4}
$$

Now using the inequality 12 in equation $(20)$, we have

$$
F\left(\rho_{W}\right) \leq \frac{1}{2}\left[1+N^{W}\right]
$$

which is the upper bound of the singlet fraction for the Werner state in terms of negativity.

We now review the status of the violation of the Bell-CHSH inequality by the Werner state. Using Eq. (7) the eigenvalues of the matrix $T_{w}^{\dagger} T_{w}$ are given by $u_{1}=u_{2}=u_{3}=\frac{\left(4 F_{w}-1\right)^{2}}{9}$, where $\left(T_{w}\right)_{n m}=\operatorname{Tr}\left(\rho_{W} \sigma_{n} \otimes \sigma_{m}\right)$ denotes the elements of the matrix $T_{w}$. The Werner state violates the Bell-CHSH inequality iff $M\left(\rho_{W}\right)>1$, where $M\left(\rho_{W}\right)$ is given by

$$
M\left(\rho_{W}\right)=2 \frac{\left(4 F_{w}-1\right)^{2}}{9}
$$

Using Eq. 16 it follows that the Werner state satisfies the Bell-CHSH inequality although it is entangled when the maximal singlet fraction $F_{w}$ lies within the range

$$
\frac{1}{2} \leq F_{w} \leq \frac{3+\sqrt{2}}{4 \sqrt{2}}
$$


The optimal teleportation fidelity in terms of $M(\rho)$ is given by

$$
f_{o p t}^{T}\left(\rho_{W}\right)=\frac{\sqrt{\frac{M\left(\rho_{W}\right)}{2}}+1}{2}
$$

Moreover, from Eqs. 17) and (24) it follows that the Werner state can be used as a quantum teleportation channel (average optimal fidelity exceeding 2/3) even without violating the Bell-CHSH inequality in the above domain.

\section{TELEPORTATION VIA THE MUNRO-JAMES-WHITE-KWIAT MAXIMALLY ENTANGLED MIXED STATE}

Munro et al. 2, 21 showed that there exist a class of states that have significantly greater degree of entanglement for a given linear entropy than the Werner state. In this section we will investigate whether the class of states introduced by Munro et al. could be used as a teleportation channel. We begin with the analytical form of the MEMS given by

$$
\rho_{M E M S}=\left(\begin{array}{cccc}
h(C) & 0 & 0 & \frac{C}{2} \\
0 & 1-2 h(C) & 0 & 0 \\
0 & 0 & 0 & 0 \\
\frac{C}{2} & 0 & 0 & h(C)
\end{array}\right)
$$

where

$$
h(C)= \begin{cases}C / 2 & C \geq \frac{2}{3} \\ 1 / 3 & C<\frac{2}{3}\end{cases}
$$

with $C$ denoting the concurrence of $\rho_{M E M S} 25$.

The form of the linear entropy is given by

$$
S_{L}= \begin{cases}\frac{8}{3}\left(C-C^{2}\right) & C \geq 2 / 3 \\ \frac{2}{3}\left(\frac{4}{3}-C^{2}\right) & C<2 / 3\end{cases}
$$

To see the performance of the MEMS state 25) as a teleportation channel, we have to calculate the fidelity of the teleportation channel. We use the result given in Eq. (9) relating the optimal teleportation fidelity and the singlet fraction of a state $\rho$. The maximal singlet fraction of the state described by the density operator $\rho_{M E M S}$ using the definition (1) is found out to be

$$
\begin{aligned}
F_{M E M S} & =\max \left\{h(C)+\frac{C}{2}, h(C)-\frac{C}{2}, \frac{1}{2}-h(C), \frac{1}{2}-h(C)\right. \\
& =h(C)+\frac{C}{2}
\end{aligned}
$$

Using Eqs. (9) and (26), the optimal teleportation fidelity is given by

$$
f_{o p t}^{T}\left(\rho_{M E M S}\right)= \begin{cases}\frac{2 C+1}{3} & C \geq 2 / 3 \\ \frac{5+3}{9} & C<2 / 3\end{cases}
$$

Now inverting the relation (27), i.e., expressing $C$ in terms of $S_{L}$, we can rewrite Eq. 29] in terms of the linear entropy $S_{L}$ as

$$
f_{o p t}^{T}\left(\rho_{M E M S}\right)= \begin{cases}\frac{2}{3}+\frac{\sqrt{2-3 S_{L}}}{3 \sqrt{2}} & 0 \leq S_{L} \leq \frac{16}{27} \\ \frac{5}{9}+\frac{\sqrt{8-9 S_{L}}}{3 \sqrt{6}} & \frac{16}{27}<S_{L} \leq \frac{8}{9}\end{cases}
$$

It follows that the MJKW 2 maximally entangled mixed state 25) can be used as a faithful teleportation channel when the mixedness of the state is less than the value $S_{L}=22 / 27$.

Note that since in specific cases of teleportation the teleportation fidelity depends upon the input states, it gives better results for some input states and worse for some other input states. But here we use the formula for average teleportation fidelity averaging over all input states. However, for specific cases of input states it is possible to perform 
a calculation for the best (or the worst) teleportation fidelity (rather than the averaged optimum) as we illustrate now. For example, if we consider the input state to be teleported is of the form

$$
\rho^{i n}=\left(\begin{array}{cc}
x & y \\
y^{*} & 1-x
\end{array}\right)
$$

and if the teleportation channel is given by $\rho_{M E M S}$, the teleported state (using the standard teleportation protocol) after performing suitable unitary transformations corresponding to the four Bell-state measurement outcomes $\left|\phi^{+}\right\rangle,\left|\phi^{-}\right\rangle,\left|\psi^{+}\right\rangle$and $\left|\psi^{-}\right\rangle$is given by (for the following two cases):

(i) For $C \geq \frac{2}{3}$

$$
\begin{array}{r}
\rho_{B_{1}}^{\text {out }}=\rho_{B_{2}}^{\text {out }}=\left(\begin{array}{ll}
\frac{x C}{2 N} & \frac{y C}{2 N} \\
\frac{y^{*} C}{2 N} & \frac{x(2-3 C)+C}{2 N}
\end{array}\right) \\
\rho_{B_{3}}^{\text {out }}=-\rho_{B_{4}}^{\text {out }}=\left(\begin{array}{cc}
\frac{(3 x-2) C+2(1-x)}{2 N_{1}} & \frac{y C}{2 N_{1}} \\
\frac{y^{*} C}{2 N_{1}} & \frac{(1-x) C}{2 N_{1}}
\end{array}\right)
\end{array}
$$

and, (ii) for $C<\frac{2}{3}$

$$
\begin{gathered}
\rho_{B_{1}^{\prime}}^{\text {out }}=\rho_{B_{2}^{\prime}}^{\text {out }}=\left(\begin{array}{cc}
\frac{x}{3 N} & \frac{y C}{2 N} \\
\frac{y^{*} C}{2 N} & \frac{1}{3 N}
\end{array}\right) \\
\rho_{B_{3}^{\prime}}^{\text {out }}=-\rho_{B_{4}^{\prime}}^{\text {out }}=\left(\begin{array}{cc}
\frac{1}{3 N_{1}} & \frac{y C}{2 N_{1}} \\
\frac{y^{*} C}{2 N_{1}} & \frac{(1-x)}{3 N_{1}}
\end{array}\right)
\end{gathered}
$$

To determine the efficiency of the teleportation channel, we calculate the distances between the input and output state using Hilbert Schmidt norm, and they are given by

(i) for $C \geq \frac{2}{3}$

$$
\begin{aligned}
D_{B_{1}} & =D_{B_{2}}=x^{2}\left(1-\frac{C}{2 N}\right)^{2}+2|y|^{2}\left(1-\frac{C}{2 N}\right)^{2} \\
& +\left[(1-x)-\frac{x(2-3 C)+C}{2 N}\right]^{2} \\
D_{B_{3}} & \left.=x-\frac{(3 x-2) C+2(1-x)}{2 N_{1}}\right]^{2}+2|y|^{2}\left(1-\frac{C}{2 N_{1}}\right)^{2} \\
& +(1-x)^{2}\left(1-\frac{C}{2 N_{1}}\right)^{2} \\
D_{B_{4}} & \left.=x+\frac{(3 x-2) C+2(1-x)}{2 N_{1}}\right]^{2}+2|y|^{2}\left(1+\frac{C}{2 N_{1}}\right)^{2} \\
& +(1-x)^{2}\left(1+\frac{C}{2 N_{1}}\right)^{2}
\end{aligned}
$$

where $N=x\left(1-\frac{C}{2}\right)+\frac{(1-x) C}{2}$ and $N_{1}=\frac{x C}{2}+(1-x)\left(1-\frac{C}{2}\right)$, and (ii) for $C<\frac{2}{3}$

$$
\begin{aligned}
D_{B_{1}^{\prime}} & =D_{B_{2}^{\prime}}=x^{2}\left(1-\frac{1}{3 N^{\prime}}\right)^{2}+2|y|^{2}\left(1-\frac{C}{2 N^{\prime}}\right)^{2} \\
& +\left[(1-x)-\frac{1}{3 N^{\prime}}\right]^{2} \\
D_{B_{3}^{\prime}} & =\left(x-\frac{1}{3 N_{1}^{\prime}}\right)^{2}+2|y|^{2}\left(1-\frac{C}{2 N_{1}^{\prime}}\right)^{2} \\
& +(1-x)^{2}\left(1-\frac{1}{3 N_{1}^{\prime}}\right)^{2} \\
D_{B_{4}^{\prime}} & =\left(x+\frac{1}{3 N_{1}^{\prime}}\right)^{2}+2|y|^{2}\left(1+\frac{C}{2 N_{1}^{\prime}}\right)^{2} \\
& +(1-x)^{2}\left(1+\frac{1}{3 N_{1}^{\prime}}\right)^{2}
\end{aligned}
$$


where where $N^{\prime}=\frac{2 x}{3}+\frac{1-x}{3}$ and $N_{1}^{\prime}=\frac{x}{3}+\frac{2(1-x)}{3}$. The teleportation fidelity $(F)$ can be easily calculated by using the formula $F=1-D$. Clearly, the fidelity depends on the input state and hence one can easily calculate the best (or worst) fidelity by choosing some particular input state. However, the puprose of the present paper is to compare the average perfomance of various teleportation channels, and to this end henceforth in this work we will deal further with average optimal teleportation fidelities only.

Next, we return to the nonlocal properties of the state $\rho_{M E M S}$. Wei et al. 21] have studied the state $\rho_{M E M S}$ from the perspective of Bell's-inequality violation. Here we focus on the parametrization of the state given by Eq. 25) and demarcate the range of concurrence where the Bell-CHSH inequality is violated. In order to use the result (13) we construct the matrix $T_{M E M S}$ as

$$
T_{M E M S}=\left(\begin{array}{ccc}
h(C)+C & 0 & 0 \\
0 & -C & 0 \\
0 & 0 & 4 h(C)-1
\end{array}\right)
$$

The eigenvalues of the matrix $\left(T_{M E M S}^{\dagger} T_{M E M S}\right)$ are given by

$$
u_{1}=(h(C)+C)^{2}, \quad u_{2}=C^{2}, \quad u_{3}=(4 h(C)-1)^{2}
$$

In accord with Eq. 26), the eigenvalues (37) take two different forms which are discussed separately below:

Case-I: $h(C)=\frac{C}{2}, \quad \frac{2}{3} \leq C \leq 1$. The eigenvalues (37) reduce to

$$
u_{1}=\frac{9 C^{2}}{4}, \quad u_{2}=C^{2}, \text { and } u_{3}=(2 C-1)^{2}
$$

When $C \geq \frac{2}{3}$, the eigenvalues can be arranged as $u_{1}>u_{2}>u_{3}$. Therefore,

$$
M\left(\rho_{M E M S}\right)=u_{1}+u_{2}=\frac{13 C^{2}}{4}
$$

One can easily see that $M\left(\rho_{M E M S}\right)>1$ when $C \geq \frac{2}{3}$, and hence, in this case the state $\rho_{M E M S}$ violates the Bell-CHSH inequality.

Case-II: $h(C)=\frac{1}{3}, \quad 0 \leq C<\frac{2}{3}$. The eigenvalues given by Eq. 377 reduce to

$$
u_{1}=\frac{(3 C+1)^{2}}{9}, \quad u_{2}=C^{2}, \text { and } u_{3}=\frac{1}{9}
$$

Now we can split the interval $0 \leq C<\frac{2}{3}$ into two sub-intervals $0 \leq C \leq \frac{1}{3}$ and $\frac{1}{3}<C<\frac{2}{3}$, where the ordering of the eigenvalues are different.

(i) when $0 \leq C \leq \frac{1}{3}$, the ordering of the eigenvalues are $u_{1}>u_{3}>u_{2}$. In this case one has

$$
M\left(\rho_{M E M S}\right)-1=u_{1}+u_{3}-1=\frac{9 C^{2}+6 C-7}{9}
$$

From Eq. 41 it is clear that $M\left(\rho_{M E M S}\right)<1$ when $0 \leq C \leq \frac{1}{3}$. Hence, the Bell-CHSH inequality is satisfied by $\rho_{M E M S}$.

(ii) when $\frac{1}{3}<C<\frac{2}{3}$, the ordering of the eigenvalues are $u_{1}>u_{2}>u_{3}$. Therefore, the expression for $\left(M\left(\rho_{M E M S}\right)-1\right)$ is given by

$$
M\left(\rho_{M E M S}\right)-1=u_{1}+u_{2}-1=\frac{2\left(9 C^{2}+3 C-4\right)}{9}
$$

From Eq. 42, it follows that $M\left(\rho_{M E M S}\right)>1$ when $\frac{\sqrt{153}-3}{18}<C<\frac{2}{3}$ and hence the state $\rho_{M E M S}$ violates the Bell-CHSH inequality. On the contrary, $M\left(\rho_{M E M S}\right) \leq 1$ when $\frac{1}{3}<C \leq \frac{\sqrt{153}-3}{18}$, and hence the state $\rho_{M E M S}$ satisfies the Bell-CHSH inequality although it is entangled. It was noticed earlier [22] that the MJKW state needs a much higher degree of entanglement to violate the Bell-CHSH inequality compared to the Werner states. Our above results revalidate this fact.

We next consider a wider class of maximally entangled mixed states as proposed by Wei et al. 21]. The general form of a two qubit density matrix comprising a mixture of the maximally entangled Bell state $\left|\Phi_{+}\right\rangle$and a mixed diagonal state is given by

$$
\rho_{G}=\left(\begin{array}{cccc}
x+\frac{\gamma}{2} & 0 & 0 & \frac{\gamma}{2} \\
0 & a & 0 & 0 \\
0 & 0 & b & 0 \\
\frac{\gamma}{2} & 0 & 0 & y+\frac{\gamma}{2}
\end{array}\right)
$$


where $a, b, x, y$ and $\gamma$ are non-negative real parameters. The normalization condition gives $x+y+\gamma+a+b=1$ The entanglement of $\rho_{G}$ is quantified by

$$
C\left(\rho_{G}\right)=\max [\gamma-2 \sqrt{a b}, 0]
$$

Therefore, the state $\rho_{G}$ is entangled only if $\gamma>2 \sqrt{a b}$. The correlation matrix for $\rho_{G}$ is given by:

$$
T_{G}=\left(\begin{array}{ccc}
\gamma & 0 & 0 \\
0 & -\gamma & 0 \\
0 & 0 & x+y+\gamma-a-b
\end{array}\right)
$$

The eigen values of the symmetric matrix $T_{G}^{\dagger} T_{G}$ are given by $v_{1}=\gamma^{2}, v_{2}=\gamma^{2}$, and $v_{3}=(x+y+\gamma-a-b)^{2}=$ $(1-2 a-2 b)^{2}$. Now, the quantity $M\left(\rho_{G}\right)$ is given by

$$
M\left(\rho_{G}\right)=\max _{i>j}\left(v_{i}+v_{j}\right)
$$

Here one is led to the following two cases. Case (i): $M\left(\rho_{G}\right)=2 \gamma^{2}$, when either $\gamma>2(a+b)-1$ and $\gamma>2 \sqrt{a b}$, for $a+b>\frac{1}{2}$ or $\gamma>1-2(a+b)$ and $\gamma>2 \sqrt{a b}$ for $a+b<\frac{1}{2}$; and Case (ii): $M\left(\rho_{G}\right)=\gamma^{2}+(1-2 a-2 b)^{2}$ when either $2(a+b)-1<\gamma<2 \sqrt{a b}$, for $a+b>\frac{1}{2}$ or $2 \sqrt{a b}<\gamma<1-2(a+b)$ for $a+b<\frac{1}{2}$. In either case the Bell-CHSH inequality is violated if $M\left(\rho_{G}\right)>1$.

Now, our task is to find the condition when the state $\rho_{G}$ could be used as a teleportation channel. Hence, we have to find the condition under which $N\left(\rho_{G}\right)>1$. In this case $N\left(\rho_{G}\right)$ is given by

$$
N\left(\rho_{G}\right)=\sqrt{v_{1}}+\sqrt{v_{2}}+\sqrt{v_{3}}=1+2(\gamma-a-b)
$$

Therefore, we have

$$
N\left(\rho_{G}\right)>1 \Rightarrow \gamma>a+b>2 \sqrt{a b}
$$

It follows from Eq.47 that

$$
f_{o p t}^{T}\left(\rho_{G}\right)=\frac{1}{2}\left[1+\frac{N\left(\rho_{G}\right)}{3}\right]=\frac{2}{3}+\frac{1}{3}(\gamma-a-b)
$$

Writing the optimal teleportation fidelity in the above form enables a useful comparison with the teleportation capacity of the Werner state. Note that for either $a=0$, or $b=0$, one has $C\left(\rho_{W}\right)=\gamma$. Hence, it follows that the average optimal teleportation fidelity of the Werner state can be written as

$$
f_{o p t}^{T}\left(\rho_{W}\right)=\frac{2}{3}+\frac{\gamma}{3}
$$

From Eqs. 49) and (50) it immediately follows that

$$
f_{o p t}^{T}\left(\rho_{G}\right)<f_{o p t}^{T}\left(\rho_{w e r n e r}\right)
$$

which shows that the Werner state performs better as a teleportation channel than the general MEMS.

\section{NON-MAXIMALLY ENTANGLED MIXED STATES AS TELEPORTATION CHANNELS}

\section{A. The Werner Derivative}

Hiroshima and Ishizaka 11] studied a particular class of mixed states - Werner derivative - obtained by applying a nonlocal unitary operator $U$ on the Werner state, i.e., $\rho_{w d}=U \rho_{W} U^{\dagger}$. The Werner derivative is described by the density operator

$$
\rho_{w d}=\frac{1-F_{w}}{3} I_{4}+\frac{4 F_{w}-1}{3}|\psi\rangle\langle\psi|
$$

where $|\psi\rangle=U\left|\Psi^{-}\right\rangle=\sqrt{a}|00\rangle+\sqrt{1-a}|11\rangle$ with $\frac{1}{2} \leq a \leq 1$. The state 52 is entangled if and only if [1]

$$
\frac{1}{2} \leq a<\frac{1}{2}\left(1+\frac{\sqrt{3\left(4 F_{w}^{2}-1\right)}}{4 F_{w}-1}\right)
$$


which futher gives a restriction on $F_{w}$ as $\frac{1}{2}<F_{w} \leq 1$.

Our aim here is to study how efficiently the Werner derivative works as a teleportation channel. To do this, let us start with the matrix $T_{w d}$ for the state $\rho_{w d}$ given by

$$
\begin{aligned}
& T_{w d}= \\
& \left(\begin{array}{ccc}
\frac{2 \sqrt{a(1-a)}\left(4 F_{w}-1\right)}{3} & 0 & 0 \\
0 & -\frac{2 \sqrt{a(1-a)}\left(4 F_{w}-1\right)}{3} & 0 \\
0 & 0 & \frac{\left(4 F_{w}-1\right)}{3}
\end{array}\right)
\end{aligned}
$$

The eigenvalues of the matrix $\left(T_{w d}^{\dagger} T_{w d}\right)$ are $u_{1}=u_{2}=\frac{4 a(1-a)\left(4 F_{w}-1\right)^{2}}{9}, u_{3}=\frac{\left(4 F_{w}-1\right)^{2}}{9}$. The Werner Derivative can be used as a teleportation channel if and only if it stisfies Eq. (8), i.e., $N\left(\rho_{w d}\right)>1$, where

$$
\begin{aligned}
N\left(\rho_{w d}\right) & =\sqrt{u_{1}}+\sqrt{u_{2}}+\sqrt{u_{3}} \\
& =\frac{\left(4 F_{w}-1\right)[1+4 \sqrt{a(1-a)}]}{3}
\end{aligned}
$$

It follows that the Werner Derivative can be used as a teleportation channel if and only if

$$
16 a^{2}-16 a+\alpha^{2}<0
$$

where $\alpha=\frac{4\left(1-F_{w}\right)}{4 F_{w}-1}$. Solving $\sqrt{56}$ for the parameter $a$, we get

$$
\frac{1}{2} \leq a<\frac{1}{2}+\frac{\sqrt{4-\alpha^{2}}}{4} \equiv \frac{1}{2}\left(1+\frac{\sqrt{3\left(4 F_{w}^{2}-1\right)}}{4 F_{w}-1}\right)
$$

Therefore, teleportation can be done faithfully via $\rho_{w d}$ when the parameter $a$ satisfies the inequality (53).

The fidelity of teleportation is given by

$$
\begin{aligned}
f_{\text {opt }}^{T}\left(\rho_{w d}\right) & =\frac{1}{2}\left[1+\frac{1}{3} N\left(\rho_{w d}\right)\right] \\
& =\frac{1}{18}\left[9+\left(4 F_{w}-1\right)(1+4 \sqrt{a(1-a)})\right]
\end{aligned}
$$

When $a=\frac{1}{2}$, the Werner derivative reduces to the Werner state, and the teleportation fidelity also reduces to that of the Werner state given by Eq. 17]. From Eq. 58, it is clear that $f_{o p t}^{T}\left(\rho_{w d}\right)$ is a decreasing function of $a$, and hence from Eq.(57), one obtains

$$
\frac{2}{3}<f_{o p t}^{T}\left(\rho_{w d}\right) \leq \frac{2 F_{w}+1}{3}
$$

Further, we can express the teleportation fidelity $f_{o p t}^{T}\left(\rho_{w d}\right)$ given in Eq. 58 in terms of linear entropy $S_{L}$ as

$$
\begin{aligned}
& f_{\text {opt }}^{T}\left(\rho_{w d}\right) \\
& \quad=\frac{9+3 \sqrt{1-S_{L}}(1+4 \sqrt{a(1-a)})}{18}, 0 \leq S_{L}<\frac{8}{9}
\end{aligned}
$$

Now we investigate whether the state $\rho_{w d}$ violates the Bell-CHSH inequality using the condition given in Eq. 13 . The real valued function $M(\rho)$ for the Werner derivative state is given by

$$
M\left(\rho_{w d}\right)=u_{2}+u_{3}=\frac{\left(1+4 a-4 a^{2}\right)\left(4 F_{w}-1\right)^{2}}{9}
$$

It follows that

$$
M\left(\rho_{w d}\right)-1=\frac{-\left(4 F_{w}-1\right)^{2}}{9}(a-\beta)(a-\gamma)
$$

where

$$
\begin{aligned}
& \beta=\frac{1}{2}\left(1-\frac{\sqrt{2\left(4 F_{w}-1\right)^{2}-9}}{4 F_{w}-1}\right) \\
& \gamma=\frac{1}{2}\left(1+\frac{\sqrt{2\left(4 F_{w}-1\right)^{2}-9}}{4 F_{w}-1}\right)
\end{aligned}
$$


For $\beta$ and $\gamma$ to be real, $\frac{3+\sqrt{2}}{4 \sqrt{2}} \leq F_{w} \leq 1$. From the expression of $\beta$ and Eq. $\sqrt[53]{ }$, it is clear that $\beta \leq \frac{1}{2} \leq a<\frac{1}{2}(1+$ $\left.\frac{\sqrt{3\left(4 F_{w}^{2}-1\right)}}{4 F_{w}-1}\right)$ as $\frac{3+\sqrt{2}}{4 \sqrt{2}} \leq F_{w} \leq 1$. Hence $a-\beta \geq 0$. Next, from the expression of $\gamma$, it follows that $\gamma \leq \frac{1}{2}\left(1+\frac{\sqrt{3\left(4 F_{w}^{2}-1\right)}}{4 F_{w}-1}\right)$. Now, we consider the following three cases separately:

Case-I: If $\gamma<a<\frac{1}{2}\left(1+\frac{\sqrt{3\left(4 F_{w}^{2}-1\right)}}{4 F_{w}-1}\right)$ and $\frac{3+\sqrt{2}}{4 \sqrt{2}}<F_{w} \leq 1$, then $M\left(\rho_{w d}\right)-1<0$. In this case the Bell-CHSH inequality is respected by the state $\rho_{w d}$ although the state is entangled there.

Case-II: If $\frac{1}{2} \leq a<\gamma$ and $\frac{3+\sqrt{2}}{4 \sqrt{2}}<F_{w} \leq 1$, then $M\left(\rho_{w d}\right)-1>0$. Thus in this range of the parameter $a$ the Bell-CHSH inequality is violated by the state $\rho_{w d}$.

Case-III: Here we consider the situation when $F_{w}=\frac{3+\sqrt{2}}{4 \sqrt{2}}$. In this case $\beta=\gamma=\frac{1}{2}$ and hence $M\left(\rho_{w d}\right) \leq 1$ holds for $\left.\frac{1}{2} \leq a<\frac{1}{2}\left(1+\frac{\sqrt{1+2 \sqrt{2}}}{2}\right)\right)$. The equality sign is achieved when $a=\beta=\gamma=\frac{1}{2}$. Therefore, in the case when $F_{w}=\frac{3+\sqrt{2}}{4 \sqrt{2}}$ the Werner derivative satisfies the Bell-CHSH inequality although it is entangled.

\section{B. A new non-maximally entangled mixed state}

We construct a two-qubit density matrix $\rho_{\text {new }}$ as a convex combination of a separable density matrix $\rho_{12}^{G}=$ $\operatorname{Tr}_{3}\left(|G H Z\rangle_{123}\right)$ and an inseparable density matrix $\rho_{12}^{W}=\operatorname{Tr}_{3}\left(|W\rangle_{123}\right)$ where $|G H Z\rangle$ and $|W\rangle$ denote the three-qubit GHZ-state 23] and the W-state 24] respectively. This construction is somewhat similar in spirit to the Werner state which is a convex combination of a maximally mixed state and a maximally entangled pure state. We exploit here the properties that the GHZ state and the W state are two qubit separable and inseparable states, respectively, when a qubit is lost from the corresponding three qubit states. By constructing this type of a non-maximally entangled mixed state, our aim is to show that it can be used as a better teleportation channel compared to the Werner derivative state.

The two-qubit state described by the density matrix $\rho_{\text {new }}$ can be explicitly written as

$$
\rho_{\text {new }}=p \rho_{12}^{G}+(1-p) \rho_{12}^{W}, \quad 0 \leq p \leq 1
$$

The matrix representation of the density matrix $\rho_{\text {new }}$ in the computational basis is given by

$$
\rho_{\text {new }}=\left(\begin{array}{cccc}
\frac{p+2}{6} & 0 & 0 & 0 \\
0 & \frac{1-p}{3} & \frac{1-p}{3} & 0 \\
0 & \frac{1-p}{3} & \frac{1-p}{3} & 0 \\
0 & 0 & 0 & \frac{p}{2}
\end{array}\right) .
$$

Since the state described by the density matrix $(65)$ is of the form

$$
\sigma=\left(\begin{array}{cccc}
a & 0 & 0 & 0 \\
0 & b & c & 0 \\
0 & c^{*} & d & 0 \\
0 & 0 & 0 & e
\end{array}\right)
$$

its amount of entanglement [25] is given by

$$
\begin{aligned}
C\left(\rho_{\text {new }}\right) & =C(\sigma)=2 \max (|c|-\sqrt{a e}, 0) \\
& =2 \max \left(\left(\frac{1-p}{3}-\sqrt{\frac{p(p+2)}{12}}\right), 0\right)
\end{aligned}
$$

Therefore, $\rho_{\text {new }}$ is entangled only if $\frac{1-p}{3}-\sqrt{\frac{p(p+2)}{12}}>0$, i.e., when $0 \leq p<0.292$.

Note that in the limiting case of $p=0$ the state $\rho_{\text {new }}$ reduces to

$$
\rho_{12}^{W}=\frac{1}{3}|00\rangle\left\langle 00\left|+\frac{2}{3}\right| \psi^{+}\right\rangle\left\langle\psi^{+}\right|
$$

where $\left|\psi^{+}\right\rangle=(|01\rangle+|10\rangle) / \sqrt{2}$. The state $\rho_{12}^{W}$ is maximally entangled since it can be put into Ishizaka and Hiroshima's [10] proposed class of MEMS. The concurrence of this state is $\frac{2}{3}$. When this state is used as a teleportation channel, the teleportation fidelity becomes $f_{\text {opt }}^{T}\left(\rho_{12}^{W}\right)=\frac{7}{9}$. Moreover, it can be checked that the state $\rho_{12}^{W}$ satisfies the Bell-CHSH inequality although it is an entangled state. 
To obtain the teleportation fidelity for the state $\rho_{\text {new }}$, we first construct the matrix $T_{n e w}$ using Eq. (7), which is given by

$$
T_{\text {new }}=\left(\begin{array}{ccc}
\frac{2(1-p)}{3} & 0 & 0 \\
0 & \frac{2(1-p)}{3} & 0 \\
0 & 0 & \frac{(4 p-1)}{3}
\end{array}\right)
$$

The eigenvalues of $\left(T_{n e w}^{\dagger} T_{n e w}\right)$ are given by $u_{1}=u_{2}=\frac{4(1-p)^{2}}{9}$ and $u_{3}=\frac{(4 p-1)^{2}}{9}$. When $p>\frac{1}{4}$, one has $N\left(\rho_{\text {new }}\right)=$ $\sqrt{u_{1}}+\sqrt{u_{2}}+\sqrt{u_{3}}=1$. Therefore, the teleportation fidelity becomes $f_{\text {opt }}^{T}\left(\rho_{\text {new }}\right)=\frac{1}{2}\left[1+\frac{1}{3} N\left(\rho_{\text {new }}\right)\right]=\frac{2}{3}$. Hence for $p>\frac{1}{4}$, the state $\rho_{\text {new }}$ cannot be used as an efficient teleportation channel since it does not overtake the classical fidelity. But when $0 \leq p<\frac{1}{4}, N\left(\rho_{\text {new }}\right)=\frac{5-8 p}{3}>1$, and hence $\rho_{\text {new }}$ can be used as an efficient teleportation channel. In this case the average optimal teleportation fidelity is given by

$$
f_{\text {opt }}^{T}\left(\rho_{\text {new }}\right)=\frac{7-4 p}{9}, \quad 0 \leq p<\frac{1}{4}
$$

and it follows that

$$
\frac{2}{3}<f_{\text {opt }}^{T}\left(\rho_{\text {new }}\right) \leq \frac{7}{9}
$$

We note here an interesting fact that the state $\rho_{\text {new }}$ cannot be used as an efficient teleportation channel when $0.25<p<0.292$ although the state is entangled there.

When $\rho_{\text {new }}$ is used as a quantum teleportation channel the mixedness of the state is given by

$$
S_{L}=\frac{2}{27}\left(8+14 p-13 p^{2}\right), \quad 0 \leq p<\frac{1}{4}
$$

Therefore, the teleportation fidelity $f_{o p t}^{T}\left(\rho_{n e w}\right)$ in terms of $S_{L}$ is given by

$$
\begin{array}{r}
f_{\text {opt }}^{T}\left(\rho_{\text {new }}\right)=\frac{7-\frac{4}{26}\left(14-\sqrt{612-702 S_{L}}\right)}{9}, \\
\frac{208}{351} \leq S_{L}<\frac{2223}{2808}
\end{array}
$$

Let us now address the question as to whether the state $\rho_{\text {new }}$ violates the Bell-CHSH inequality. We again calculate the real valued function $M\left(\rho_{\text {new }}\right)$ for the state $\rho_{\text {new }}$ for the two following cases separately.

Case-I: When $0 \leq p<\frac{1}{2}, M\left(\rho_{\text {new }}\right)=u_{1}+u_{2}=\frac{8+8 p^{2}-16 p}{9}$. Substituting the values of $p$ in the above range it is easy to see that $M\left(\rho_{\text {new }}\right) \leq 1$, i.e., the Bell-CHSH inequality is satisfied.

Case-II: When $\frac{1}{2} \leq p \leq 1, M\left(\rho_{\text {new }}\right)=u_{1}+u_{3}=\frac{20 p^{2}-16 p+5}{9}$. It easily follows that for the given range of values of $p$, one has $M\left(\rho_{\text {new }}\right) \leq 1$.

Therefore, we conclude that in any case (i.e. $0 \leq p \leq 1$ ), the constructed state $\rho_{\text {new }}$ does not violate the Bell-CHSH inequality although it is entangled for $0 \leq p<0.292$.

\section{COMPARISON OF TELEPORTATION FIDELITIES FOR DIFFERENT MIXED STATES}

In the earlier sections we have studied the teleportation capacities of various maximally as well as non-maximally entangled mixed channels. It would be interesting now to actually compare their performance in terms of the average optimal fidelities corresponding to their respective magnitudes of entanglement, mixedness, and also in relation to their nonlocality properties manifested by the violations of the Bell-CHSH inequality. Let us first compare the two MEMS states, viz., the Werner state and the MJKW state, whose average optimal teleportation fidelities in terms of their respective concurrences are given by (Eqs. 18) and (29)),

$$
\begin{array}{r}
f_{o p t}^{T}\left(\rho_{W}\right)=\frac{2+C\left(\rho_{W}\right)}{3} \\
f_{\text {opt }}^{T}\left(\rho_{M E M S}\right)= \begin{cases}\frac{2 C+1}{3} & C \geq 2 / 3 \\
\frac{5+3}{9} & C<2 / 3\end{cases}
\end{array}
$$




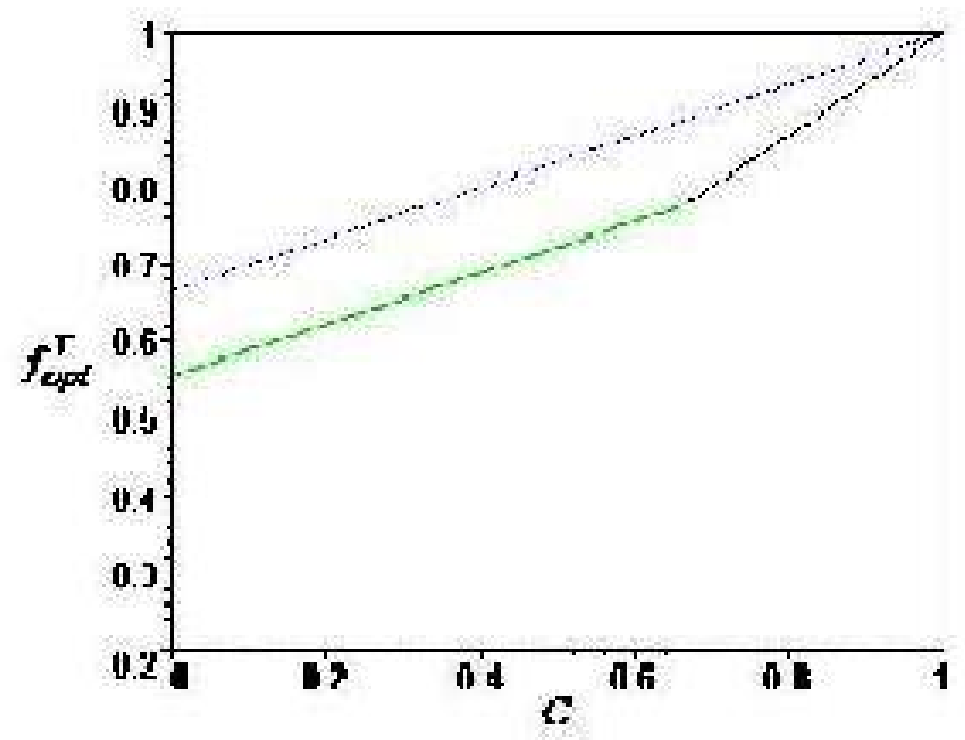

FIG. 1: (Coloronline) The average optimal teleportation fidelities for the channels $\rho_{W}$ (dotted line) and $\rho_{M E M S}$ (full line) are plotted with respect to their respective magnitudes of entanglement $C$. Note that $\rho_{M E M S}$ performs as a quantum channel only for $C>1 / 3$.

In Fig.1 we plot $f_{o p t}^{T}$ versus $C$ respectively for these two MEMS states. One can see that the Werner state performs better as a teleportation channel compared to the MJKW state for any given amount of entanglement. Note further, that the MJKW state is useful for teleportation $\left(f_{o p t}^{T}\left(\rho_{M E M S}\right)>2 / 3\right)$ only when $C>1 / 3$, whereas the Werner state is able to serve as a quantum teleportation channel for any amount of its entanglement.

Next, we compare the efficiency of teleportation of the two MEMS states with respect to their nonlocality properties. The average teleportation fidelities corresponding to the Werner state and the MJKW state are plotted versus the function $M(\rho)$ in Fig.2. Since $M(\rho)>1$ signifies the violation of the Bell-CHSH inequality, it can be seen from the figure that the MJKW state violates the Bell-CHSH inequality and simultaneously performs as a teleportation channel in a certain region of parameter space, as outlined in Section III. This is in contrast to the behaviour of the Werner state which satisfies the Bell-CHSH inequality (in the parameter space obtained in Section II) but yet performs as a quantum teleportation channel. Next, in Fig.3 we present a comparison of the Werner state and the generalized MEMS state $\rho_{G}$ by plotting respecively their average teleportation fidelities versus the function $M(\rho)$. We observe that the Werner State and the general MEMS state are both useful for teleportation whether they violate the Bell-CHSH inequality or not. But, the Werner state always performs better as as a teleportation channel compared to the general MEMS $\rho_{G}$, except at the value of $M(\rho)=1.7672$ where the teleportation fidelities for both the states are the same. The result that Werner states perform better as teleportation channels compared to the other MEMS class of states can also be understood in terms of their respective negativities $N$, i.e., $N^{M J W K}<N^{W}$ [21].

The relationship between the mixedness of a channel and its ability to perform quantum teleportation is one of the focal points of investigation in this paper. For this purpose, let us recall the expressions $\sqrt{19}, \sqrt{30}),(60)$ and $(73)$ for the teleportation fidelities in terms of the linear entropy for all the four types of states studied by us:

$$
\begin{aligned}
& f_{\text {opt }}^{T}\left(\rho_{\text {new }}\right)=\frac{7-\frac{4}{26}\left(14-\sqrt{612-702 S_{L}}\right)}{9} \\
& \frac{208}{351} \leq S_{L}<\frac{2223}{2808} \\
& f_{\text {opt }}^{T}\left(\rho_{w d}\right)=\frac{9+3 \sqrt{1-S_{L}}(1+4 \sqrt{a(1-a)})}{18}, \\
& 0 \leq S_{L}<\frac{8}{9}
\end{aligned}
$$




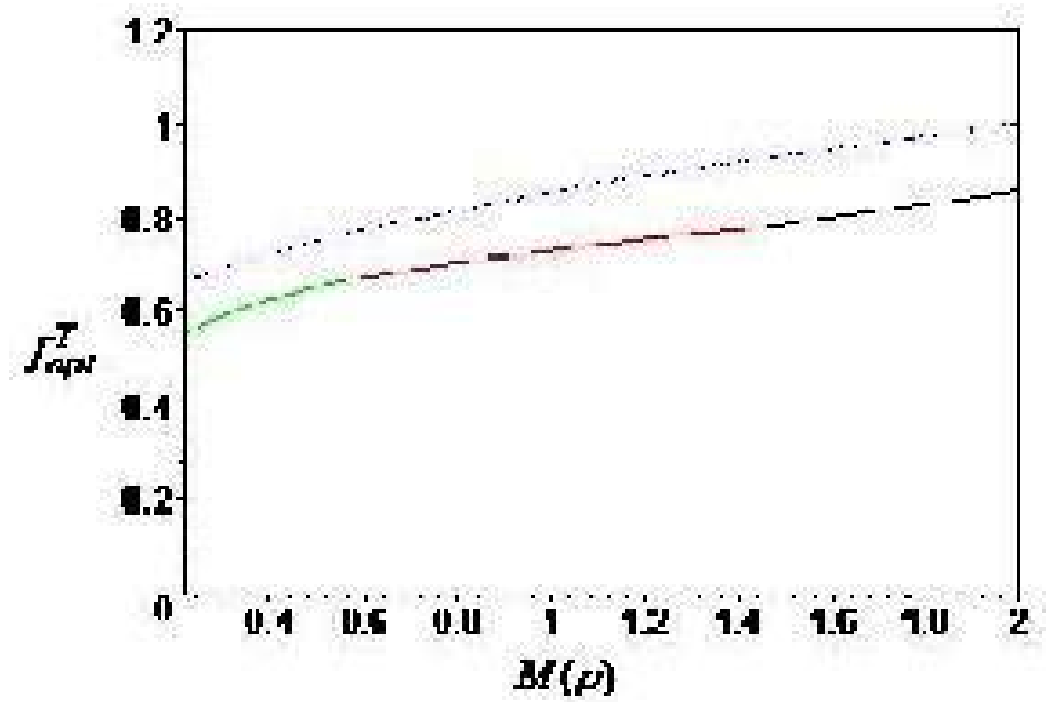

FIG. 2: (Coloronline) The average optimal teleportation fidelities for the channels $\rho_{W}$ (dotted line) and $\rho_{M E M S}$ (full line) are plotted with respect to the quantity $M(\rho)$ indicating the nonlocal property of the channel. $M(\rho)>1$ signifies the violation of the Bell-CHSH inequality.

$$
\begin{aligned}
& f_{o p t}^{T}\left(\rho_{M E M S}\right)= \begin{cases}\frac{2}{3}+\frac{\sqrt{2-3 S_{L}}}{3 \sqrt{2}} & 0 \leq S_{L} \leq \frac{16}{27} \\
\frac{5}{9}+\frac{\sqrt{8-9 S_{L}}}{3 \sqrt{6}} & \frac{16}{27} \leq S_{L} \leq \frac{8}{9}\end{cases} \\
& f_{o p t}^{T}\left(\rho_{W}\right)=\frac{1+\sqrt{1-S_{L}}}{2}, \quad 0 \leq S_{L}<\frac{8}{9}
\end{aligned}
$$

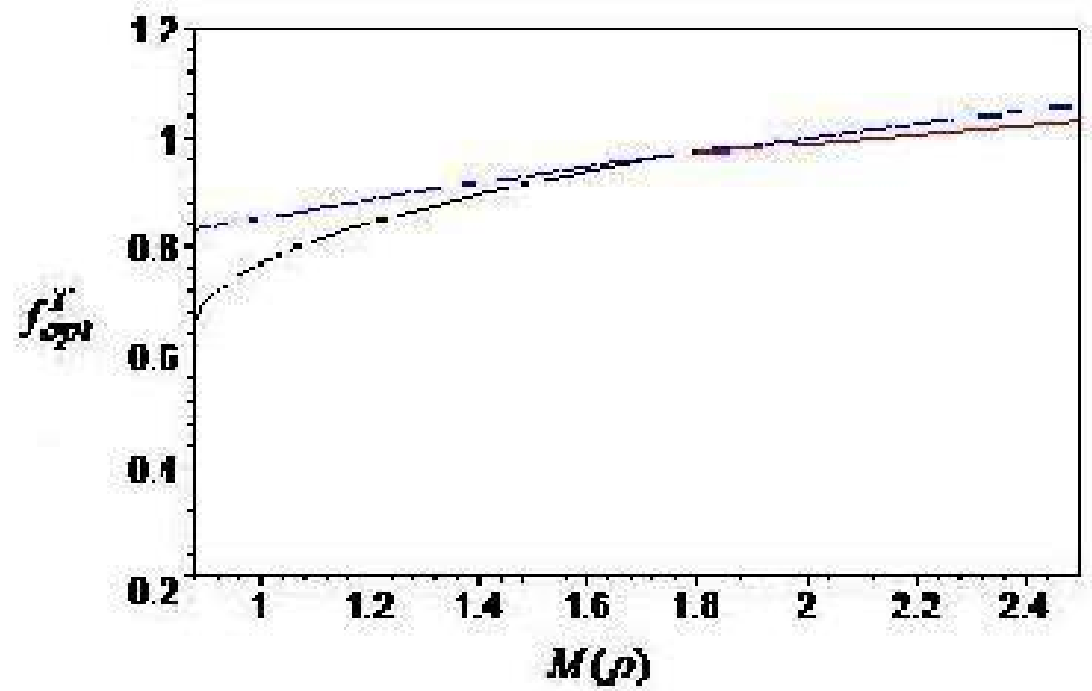

FIG. 3: (Coloronline) The average optimal teleportation fidelities for the channels $\rho_{W}$ (dotted line) and $\rho_{G}$ (full line) are plotted with respect to the quantity $M(\rho)$ indicating the nonlocal property of the channel. $M(\rho)>1$ signifies the violation of the Bell-CHSH inequality.

We first consider the comparison between the two maximally entangled states, viz., the Werner state[1] $\rho_{W}$ and the MJKW state 2] $\rho_{M E M S}$. From the above expressions of $f_{o p t}^{T}$ for these two states it follows that $f_{o p t}^{T}\left(\rho_{W}\right)=$ $f_{o p t}^{T}\left(\rho_{M E M S}\right)$ only for $S_{L}=0$. For all finite degrees of mixedness, $f_{o p t}^{T}\left(\rho_{W}\right)>f_{o p t}^{T}\left(\rho_{M E M S}\right)$. The two respective 
fidelities are plotted versus the linear entropy in Fig.4. The MJKW state can be used as a quantum teleportation channel only when its mixedness is less than $S_{L}<22 / 27$. Although both these states could perform as quantum teleportation channels for a range of values of mixedness, one sees that the Werner state outperforms the MJKW state for all finite values of mixedness even though the latter is more entangled for specific values of linear entropy 21. This is an interesting result showing that all the entanglement of the MJKW class of states is less useful as a resource for teleportation.

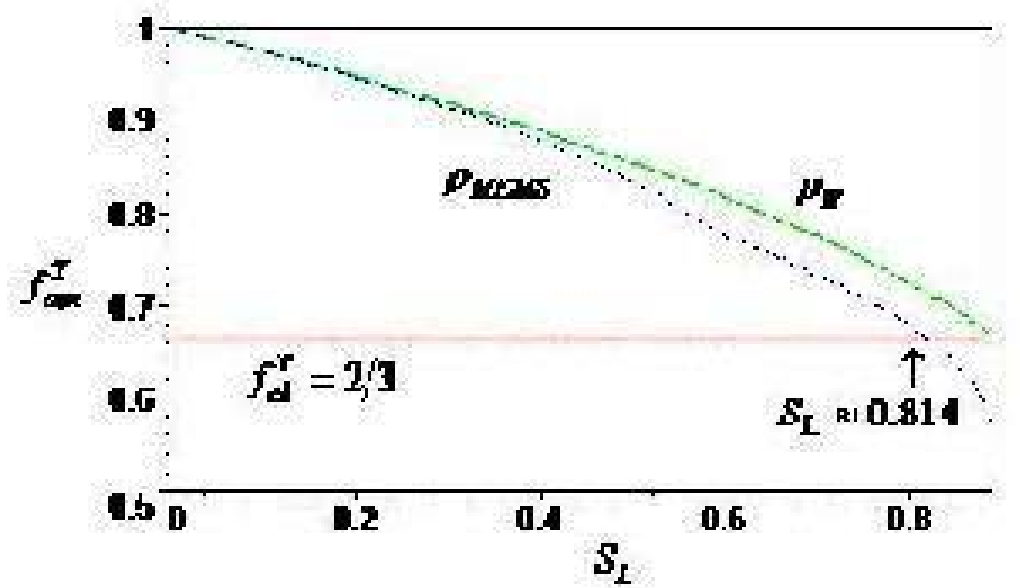

FIG. 4: (Coloronline) The average optimal teleportation fidelities for the channels $\rho_{W}$ and $\rho_{M E M S}$ are plotted with respect to the linear entropy $S_{L}$. The horzintal line represents the maximum classical fidelity.

Let us now devote our attention to the comparison of the two non-maximally entangled mixed states $\rho_{w d}$ and $\rho_{n e w}$ that we have studied in this paper. To address the issue as to which of $\rho_{w d}$ and $\rho_{n e w}$ is more efficient as resource for teleportation, we derive ranges for the parameters for which the condition $N\left(\rho_{\text {new }}\right)>N\left(\rho_{w d}\right)$ holds such that the teleportation fidelity via the channel $\rho_{\text {new }}$ will be greater than the teleportation fidelity via $\rho_{w d}$. Here we make use of the relationship between the teleportation fidelity and the quantity $N(\rho)$ [16] given by Eq. 60. In the previous sections we have calculated $N\left(\rho_{w d}\right)$ and $N\left(\rho_{\text {new }}\right)$, and their expressions are given by

$$
\begin{gathered}
N\left(\rho_{w d}\right)=\frac{\left(4 F_{w}-1\right)(1+4 \sqrt{a(1-a)})}{3}, \quad \frac{1}{2}<F_{w} \leq 1 \\
N\left(\rho_{\text {new }}\right)=\frac{5-8 p}{3}, \quad 0 \leq p<\frac{1}{4}
\end{gathered}
$$

where the parameter $a$ lies within the range specified in Eq. (53). The state $\rho_{\text {new }}$ performs better as a quantum channel for teleportation compared to the state $\rho_{w d}$ only when $N\left(\rho_{\text {new }}\right)>N\left(\rho_{w d}\right)$, from which using Eqs. (76) and (77) it follows that

$$
p<1-\left(\frac{1+2 F_{w}}{4}+\frac{\left(4 F_{w}-1\right) \sqrt{a(1-a)}}{2}\right)
$$

One can easily verify that the condition (78) on the value of $p$ is compatible with the upper bound on $p$ in Eq. (77). However, consistency with the lower bound $(p>0)$ imposes the following conditions on the parameters $F_{w}$ and $a$ :

$$
\begin{gathered}
\frac{1}{2}+\frac{\sqrt{\left(F_{w}+1\right)\left(3 F_{w}-2\right)}}{4 F_{w}-1}<a<\frac{1}{2}\left(1+\frac{\sqrt{3\left(4 F_{w}^{2}-1\right)}}{4 F_{w}-1}\right) \\
F_{w}>\frac{2}{3}
\end{gathered}
$$

Therefore, when the parameters $F_{w}, a$ and $p$ satisfy the relations given in Eqs. 78 and $(79)$, one has $f_{\text {opt }}^{T}\left(\rho_{\text {new }}\right)>$ $f_{o p t}^{T}\left(\rho_{w d}\right)$. 


\begin{tabular}{|c|c|c|c|c|}
\hline$F_{w}$ & $a$ & $p$ & $f_{\text {opt }}^{T}\left(\rho_{w d}\right)$ & $f_{\text {opt }}^{T}\left(\rho_{\text {new }}\right)$ \\
\hline 0.96 & 0.962437 & 0.000006 & 0.777775 & 0.777775 \\
\hline & 0.962490 & 0.000189 & 0.777694 & 0.777694 \\
\hline & 0.970142 & 0.028321 & 0.765190 & 0.765191 \\
\hline & 0.970144 & 0.028320 & 0.765187 & 0.765191 \\
\hline & & & & \\
\hline 0.97 & 0.964903 & 0.000003 & 0.777776 & 0.777776 \\
\hline & 0.964990 & 0.000320 & 0.777635 & 0.777636 \\
\hline & 0.978256 & 0.054980 & 0.753341 & 0.753342 \\
\hline & 0.978258 & 0.054980 & 0.753338 & 0.753342 \\
\hline & & & & \\
\hline 0.98 & 0.967213 & 0.000004 & 0.777775 & 0.777776 \\
\hline & 0.967290 & 0.000290 & 0.777644 & 0.777649 \\
\hline & 0.985910 & 0.087920 & 0.738701 & 0.738702 \\
\hline & 0.985913 & 0.087938 & 0.738693 & 0.738694 \\
\hline & & & & \\
\hline 0.99 & 0.969377 & 0.000004 & 0.777776 & 0.777776 \\
\hline & 0.969390 & 0.000056 & 0.777752 & 0.777753 \\
\hline & 0.993147 & 0.132901 & 0.718710 & 0.718711 \\
\hline & 0.993149 & 0.13291 & 0.718703 & 0.718707 \\
\hline
\end{tabular}

TABLE I: Comparison of teleportation fidelities when $\rho_{w d}$ violates the Bell-CHSH inequality while $\rho_{\text {new }}$ satisfies it.

In order to understand better the comparitive performance of the two NMEMS channels, let us rephrase our above arguments. For $\rho_{\text {new }}$ to perform better than $\rho_{w d}$, we must have $f_{o p t}^{T}\left(\rho_{\text {new }}\right)>f_{\text {opt }}^{T}\left(\rho_{w d}\right)$, which in turn implies that $N\left(\rho_{\text {new }}\right)>N\left(\rho_{w d}\right)$ since $N(\rho)$ is related to the teleportation fidelity of a channel by $\left.f_{o p t}^{T}(\rho)=\frac{1}{2}\left[1+\frac{N(\rho)}{3}\right]\right)$. We have shown that the relation $f_{o p t}^{T}\left(\rho_{\text {new }}\right)>f_{o p t}^{T}\left(\rho_{w d}\right)$ holds true only when the inequality 780 is satisfied with appropriate choices of $a$ and $F_{w}$ which are the parameters of the state $\rho_{w d}$, and $p$ which is a parameter of our constructed state $\rho_{\text {new }}$ depends. The message that one obtains from the above calculations is that similar to the case of maximally entangled mixed states (Werner and MJKW), one can also construct different classes of non-maximally entangled mixed states where one class can outperform another as a teleportation channel depending upon the chosen parameters of the states.

Next we consider the situation in which the $\rho_{w d}$ violates the Bell-CHSH inequality but $\rho_{\text {new }}$ satisfies it. In this case let us see if the teleportation fidelity $f_{o p t}^{T}\left(\rho_{w d}\right)$ could still be less than the teleportation fidelity $f_{\text {opt }}^{T}\left(\rho_{\text {new }}\right)$. We have earlier shown in Section IV that $\rho_{\text {new }}$ satisfies the Bell-CHSH inequality, and we have also derived the ranges for the parameters $a$ and $F_{w}$ for which $\rho_{w d}$ violates the inequality. Combining these conditions with the requirements (78) and $\sqrt{79}$, we obtain several possible values for the parameters $a, F_{w}$ and $p$ for which $f_{o p t}^{T}\left(\rho_{\text {new }}\right)>f_{\text {opt }}^{T}\left(\rho_{w d}\right)$. These are listed in Table-I.

We now present together the comparitive performance of all the four entangled mixed states that we have considered in this paper. We obtain the average optimal teleportation fidelities of $\rho_{W}, \rho_{M E M S}, \rho_{w d}$ and $\rho_{\text {new }}$ in terms of their linear entropies. Here we clearly address the question as to how they compete as teleportation resources for specified values of mixedness. The expressions for the teleportation fidelities of $\rho_{W}, \rho_{M E M S}$ and $\rho_{\text {new }}$ are provided explicitly in terms of the linear entropy $S_{L}$ in Eqs. 75 . But for the state $\rho_{w d}$ we first obtain $F_{w}$ for a given $S_{L}$ using the relation $F_{w}=\frac{1+3 \sqrt{1-S_{L}}}{4}$. We then select a couple of values for the parameter $a$ which lies in the range given in Eq. $\left.\sqrt{79}\right)$. Since the mixedness of the state $\rho_{w d}$ does not depend on the parameter $a$, there exists a family of states $\rho_{w d}$ for a given mixedness. Finally the corresponding value of $f_{o p t}^{T}\left(\rho_{w d}\right)$ is computed using the relation provided in Eq. 775 . Our results are presented in Table-II.

The values for the linear entropy for which the corresponding teleportation fidelities are displayed in Table-II are chosen such that all the salient features of our results that we wish to highlight are revealed in the range chosen. As expected, the MEMS states perform better as teleportation channels in general compared to the NMEMS states, with the Werner state giving rise to higher teleportation fidelity for all values of mixedness. The comparison between the two NMEMS states is affected by the fact that for a given $S_{L}$ there exists a family of states $\rho_{w d}$ corresponding to different admissible values of $a$. In this range some $\rho_{w d}$ states perform better compared to $\rho_{n e w}$, but the situation may be reversed for a different value of $a$ corresponding to the same value of mixedness. Moreover, since mixedness for $\rho_{w d}$ does not depend on $a$, there exist some values of $a$ for which $\rho_{w d}$ even outperforms the MJKW state $\rho_{M E M S}$, as displayed in the Table. 


\begin{tabular}{|c|c|c|c|c|c|}
\hline$S_{L}$ & $a$ & $f_{\text {opt }}^{T}\left(\rho_{W}\right)$ & $f_{\text {opt }}^{T}\left(\rho_{M E M S}\right)$ & $f_{\text {opt }}^{T}\left(\rho_{w d}\right)$ & $f_{\text {opt }}^{T}\left(\rho_{\text {new }}\right)$ \\
\hline 0.593 & 0.82 & 0.818983 & 0.777625 & 0.769726 & 0.777603 \\
\hline 0.593 & 0.95 & 0.818983 & 0.777625 & 0.6990218 & 0.777603 \\
\hline 0.600 & 0.80 & 0.816228 & 0.774982 & 0.774064 & 0.774581 \\
\hline 0.600 & 0.93 & 0.816228 & 0.774982 & 0.712989 & 0.774581 \\
\hline 0.62 & 0.77 & 0.808221 & 0.767251 & 0.775686 & 0.765728 \\
\hline 0.62 & 0.90 & 0.808221 & 0.767251 & 0.726029 & 0.765728 \\
\hline 0.64 & 0.74 & 0.800000 & 0.759226 & 0.775454 & 0.756516 \\
\hline 0.64 & 0.85 & 0.800000 & 0.759226 & 0.742823 & 0.756516 \\
\hline 0.66 & 0.70 & 0.791548 & 0.750871 & 0.775321 & 0.746896 \\
\hline 0.66 & 0.92 & 0.791548 & 0.750871 & 0.702642 & 0.746896 \\
\hline
\end{tabular}

TABLE II: Comparison of teleportation fidelities for different MEMS and NMEMS channels for a given mixedness.

\section{CONCLUSIONS}

To summarize, in this paper we have studied the efficiency of maximally (MEMS) and non-maximally (NMEMS) entangled mixed states as resources for teleportation. Since not every mixed entangled state is useful for teleportation [16], we have addressed here the following questions. Is every maximally entangled mixed state useful for teleportation ? We answer this question in the negative by providing the example of the maximally entangled MJKW [2] class of states which is not useful for teleportation when its mixedness exceeds a certain bound. Another question that we have investigated here is the relation between the amount of entanglement for a state and its efficiency as a teleportation channel. Our results show that a state which is less entangled for a given degree of mixedness, e.g., the Werner state [1, could act as a more efficient teleportation channel compared to a state that is more entangled, e.g., the MJKW state.

One of our motivations here has been to compare the performance of mixed entangled states as teleportation channels for a specified amount of mixedness on one hand, and the amount of entanglement on the other. We have considered two specific well-known MEMS, viz., the Werner state and the MJWK state, and obtained their average teleportation fidelities in terms of their respective concurrences, and also in terms of their respective linear entropies. In spite of the fact that both these states fall in the category of maximally entangled mixed states, we find that one of the them, viz., the Werner state, outperforms the other, viz., the Munro state for either any fixed degree of mixedness, or any specified magnitude of entanglement. We have further considered two more class of mixed states that are not maximally entangled (NMEMS). We have shown that the Werner derivative [1] can act as an efficient quantum teleportation channel (with its average teleportation fidelity exceeding the classical bound of 2/3) in certain ranges of parameter values. We then ask the question as to whether there exist other class of NMEMS that could outperform the Werner derivative as a teleportation resource. We answer this question in the affirmative by constructing a new non-maximally entangled mixed state which is a convex combination of a separable state and an entangled state.

We have further investigated the issue as to whether the nonlocal properites of quantum states, as characterized by the violation of local inequalities, have any bearing on the ability of mixed states to teleport efficiently [4. For MEMS states, our analysis shows that the Werner state satisfies the Bell-CHSH inequality and yet performs as a quantum teleportation channel in a certain range of parameter space. We then ask the specific question: is there any NMEMS state which does not violate the Bell-CHSH inequality but is still useful for teleportation? In this context we first derive the conditions on the parameters for which the Werner derivative state [11 satisfies the criterion of nonlocality by violating the Bell-CHSH inequality. We then show that our constructed new NMEMS state could perform as a quantum teleportation channel in spite of satisfying the Bell-CHSH inequality. Moreover, our constructed state yields a higher teleportation fidelity compared to the Werner derivative even for a range of parameter values where the latter violates the Bell-CHSH inequality. We conclude by noting that for both maximally and non-maximally entangled mixed states neither the magnitude of entanglement nor the violation of local inequalities may be good indicators of their ability to perform quantum information processing tasks such as teleportation.

[1] R. F. Werner, Phys. Rev. A 40, 4277 (1989).

[2] W. J. Munro, D. F. V. James, A. G. White, P. G. Kwiat, Phys. Rev. A 64, 030302 (2001).

[3] C. H. Bennett, G. Brassard, C. Crepeau, R. Jozsa, A. Peres, W. K. Wootters, Phys. Rev. Lett. 70, 1895 (1993).

[4] S. Popescu, Phys. Rev. Lett. 72, 797 (1994).

[5] S. Massar, S. Popescu; Phys. Rev. Lett. 74, 1259 (1995). 
[6] N. Gisin, Phys. Lett. A210, 157 (1996).

[7] M. Horodecki, P. Horodecki, R. Horodecki, Phys. Rev. A 60, 1888(1999).

[8] V. Buzek and M. Hillery, Phys. Rev. A54, 1844 (1996).

[9] S. Adhikari, N. Ganguly, I. Chakrabarty, B. S. Choudhury, J. Phys. A: Math. Theor. 41, 415302 (2008).

[10] S. Ishizaka, T. Hiroshima, Phys. Rev. A 62, 022310 (2000).

[11] T. Hiroshima, S. Ishizaka, Phys. Rev. A 62, 044302 (2000).

[12] J. F. Clauser, M. A. Horne, A. Shimony and R. A. Holt, Phys. Rev. Lett. 23, 880 (1969).

[13] J. Lee and M. S. Kim, Phys. Rev. Lett. 84, 4236 (2000).

[14] W. K. Wootters, Phys. Rev. Lett. 80, 2245 (1998).

[15] S. Bose, V. Vedral, Phys. Rev. A 61, 040101(2000).

[16] R. Horodecki, M. Horodecki, P. Horodecki, Phys. Lett. A 222, 21 (1996).

[17] F. Verstraete and H. Verschilde, Phys. Rev. A 66, 022307 (2002).

[18] R. Horodecki and M. Horodecki, Phys. Rev. A54, 1838 (1996).

[19] P. Badziag, M. Horodecki, P. Horodecki, R. Horodecki, Phys. Rev. A 62, 012311 (2000).

[20] L. Mista Jr., R. Filip, J. Fiurasek, Phys. Rev. A 65, 062315 (2002).

[21] T-C. Wei, K. Nemoto, P. M. Goldbart, P. G. Kwiat, W. J. Munro, and F. Verstraete, Phys. Rev. A 67, 022110 (2003).

[22] W. J. Munro, K. Nemoto and A. G. White, J. Mod. Opt. 48, 1239 (2001).

[23] D. M. Greenberger, M. Horne and A. Zeilinger, in "Bell's theorem, Quantum theory, and conceptions of the Universe", ed. M. Kafatos (Kluwer, Dordrecht, 1989), p 69; D. Bouwmeester, et al.. Phys. Rev. Lett. 82, 1345 (1999).

[24] A. Zeilinger, M. A. Horne and D. Greenberger, in NASA Conf. Publ. No. 3135 (Code NTT, Washington DC, 1997); W. Dür, G. Vidal and J. I. Cirac, Phys. Rev. A 62, 062314 (2000).

[25] D. Bru $\beta$, C. Macchiavello, Found. Phys. 33 (11), 1617 (2003). 